\title{
Consequences of cell-to-cell P-glycoprotein transfer on acquired multidrug resistance in breast cancer: a cell population dynamics model
}

\author{
Jennifer Pasquier ${ }^{1 \dagger}$, Pierre Magal ${ }^{2 \dagger}$, Céline Boulangé-Lecomte ${ }^{1}$, Glenn Webb ${ }^{3}$, Frank Le Foll ${ }^{1 *}$
}

\begin{abstract}
Background: Cancer is a proliferation disease affecting a genetically unstable cell population, in which molecular alterations can be somatically inherited by genetic, epigenetic or extragenetic transmission processes, leading to a cooperation of neoplastic cells within tumoural tissue. The efflux protein P-glycoprotein (P-gp) is overexpressed in many cancer cells and has known capacity to confer multidrug resistance to cytotoxic therapies. Recently, cell-tocell P-gp transfers have been shown. Herein, we combine experimental evidence and a mathematical model to examine the consequences of an intercellular P-gp trafficking in the extragenetic transfer of multidrug resistance from resistant to sensitive cell subpopulations.

Methodology and Principal Findings: We report cell-to-cell transfers of functional P-gp in co-cultures of a P-gp overexpressing human breast cancer MCF-7 cell variant, selected for its resistance towards doxorubicin, with the parental sensitive cell line. We found that P-gp as well as efflux activity distribution are progressively reorganized over time in co-cultures analyzed by flow cytometry. A mathematical model based on a Boltzmann type integropartial differential equation structured by a continuum variable corresponding to P-gp activity describes the cell populations in co-culture. The mathematical model elucidates the population elements in the experimental data, specifically, the initial proportions, the proliferative growth rates, and the transfer rates of P-gp in the sensitive and resistant subpopulations.
\end{abstract}

Conclusions: We confirmed cell-to-cell transfer of functional P-gp. The transfer process depends on the gradient of P-gp expression in the donor-recipient cell interactions, as they evolve over time. Extragenetically acquired drug resistance is an additional aptitude of neoplastic cells which has implications in the diagnostic value of P-gp expression and in the design of chemotherapy regimens.

Reviewers: This article was reviewed by Leonid Hanin, Anna Marciniak-Czochra and Marek Kimmel.

\section{Introduction}

\section{Current view on cancer}

In essence, cancer is a proliferation disease affecting a genetically unstable cell population. The somatic mutation theory, which is the prevailing paradigm, stipulates that cancer arises from a stepwise accumulation of changes in genes that progressively drives subclonal neoplastic cells to evolve independently from the others, escaping from proliferation control and competing for

\footnotetext{
* Correspondence: frank.lefoll@univ-lehavre.fr

† Contributed equally

'Laboratory of Ecotoxicology UPRES EA 3222, IFRMP 23, University of Le

Havre, 76058 Le Havre cedex France

Full list of author information is available at the end of the article
}

space and resources and, finally, to kill the host. The key feature is that the cancer-promoting changes are intrinsic molecular events that are $i$ ) inheritable by daughter cells and $i$ ) selectable, in the sense that they confer selective advantage in the cell population environment $[1,2]$. The essential set of aptitudes that a cell must acquire to become malignant includes selfsufficiency of growth factors, resistance to death signals, evasion from apoptosis, limitless replicative potential, sustained angiogenesis, and proclivity for tissue invasion and metastasis [2]. It is also widely recognized that genetic instability results in the creation of diverse daughter cells, capable of interaction with the cell

\section{C) Biomed Central}


microenvironment, conferring to the tumour the properties of a heterogeneous tissue [3].

Within such tumour tissue, molecular alterations are considered to be somatically inherited by genetic, epigenetic (traits that are not dependent on the primary sequence of DNA) or extragenetic (post-translational protein modifications that initiate and support positive feedback loops) transmission processes [1], leading to a cooperation of neoplastic cells [4].

\section{Role of P-glycoprotein in breast cancer}

Despite decades of research, breast cancer remains a major public health issue. Worldwide, breast cancer represents $23 \%$ of overall female cancers and accounts for 40,000 deaths each year in the United States alone. Currently, the lifetime risk of developing a breast cancer for women born in the United States is 1 in 8 . For metastatic forms of breast cancers, the 5 -year relative survival is only $26 \%$ [5] and complete remission has been estimated as low as $3.1 \%$ after this period [6]. Response to first-line chemotherapies span from 30 to $65 \%$ and are followed, after a period varying from 6 to 10 months, by disease progression [7]. In fact, resistance to chemotherapy is believed to cause treatment failure in $90 \%$ of metastatic breast cancer patients [8].

Resistance to chemotherapy is related to the overall mechanisms that are involved in a decrease of drug efficacy against tumours [9]. Among these factors, proteins lowering the intracellular concentration of chemotherapeutics and belonging to the ATP-binding Cassette (ABC) transporters are well-known for their specific responsibility [10]. These membrane proteins are characterized by their ability to efflux a large panel of both chemically and functionally unrelated compounds comprising potent cytotoxics currently used in chemotherapeutic treatments. Consequently, tumours overexpressing this type of energy-dependent pump have been very early identified on the basis of their Multi-Drug Resistance (MDR) phenotype [11]. The P-glycoprotein (P-gp) was the first drug-efflux protein characterized [12]. With the growing number of molecularly cloned $A B C$ transporters (48 different genes up to now have been identified in the human genome), a rational nomenclature have been proposed. Meta-analysis [13] or immunochemistry studies [14] have determined that approximately $40 \%$ of all breast cancer tumours express $A B C B 1 / M D R 1$ coded P-gp. $A B C B 1 / M D R 1$ gene expression has a prognostic value for cell resistance to anticancer drugs $[15,16]$ and for treatment failure [17].

\section{Genetic versus extragenetic resistance transmission}

Until now, the development of multi-drug resistance in neoplastic cells was explained as the consequences of two main mechanisms. On one hand, cells natively expressing drug-efflux proteins retain their phenotype throughout the process of malignant transformation. On the other hand, in non-expressing cells, chemotherapeutics have been shown first to induce P-gp expression and, second, to exert a selection of resistant cells during the course of chemotherapy [18-21]. Therefore, P-gp induction was considered to only depend on the cell type and/or the previous history of exposure to cytotoxics. Biomathematical models have been proposed to describe the kinetics of P-gp induction as a function of tissue cytotoxic concentration [22] with the objective of adapting the time course of drug administration to overcome as much as possible multidrug resistance.

Recently however, extragenetic transmissions of multidrug resistance have been reported. These unexpected events all involve direct transfers of P-gp to $A B C B 1 /$ $M D R 1$ non-expressing recipient cells from various multidrug resistance donors, namely adherent cell lines in vitro and in vivo [23], stromal cells isolated from patients suffering of ovarian cancers [24] and lymphoblastic leukemia cells in suspension [25]. These findings actually add a new modality of resistance appearance, and possibly spreading, in a population of tumour cells. Theoretically, cell-to-cell P-gp transfers in conditions of antineoplastic treatment could confer a significant advantage to sensitive cells, keeping them alive long enough to produce their own P-gp copies, under the induction process, and thus, to resist chemotherapy. However, the real dynamics of extragenetic acquired resistance in a growing population of cancer cells has remained, until now, unknown. Herein, we propose a biomathematical model, derived from a previous analysis [26], supported by experimental parameters and involving processes of $i$ ) cell proliferation and death, ii) P-gp induction and degradation and iii) cell-to-cell P-gp transfer, to help answer this question. A parallel study in progress will present experimental data on mechanics of transfer [27]. The present model was supplied by new data obtained in vitro from MCF-7 human breast adenocarcinoma, a cell line that has previously been shown to be capable of intercellular P-gp transfer [23]. The main objectives of the present work were to investigate the influence of both cancer cell line intrinsic factors (growth rate, initial proportions of sensitive and resistant cells, and P-gp transfer rate), as well as treatment management parameters (i.e. cycles of drug administration), on overall multidrug resistance, both genetically and extragenetically transmitted.

\section{Results}

\section{MCF-7 proliferation and resistance}

In 2005, Levchenko et al. have shown that several drug sensitive cell lines, including the well-established human breast adenocarcinoma cells MCF-7, are able to acquire 
multidrug resistance extragenetically, via a direct intercellular transfer of the resistance protein P-gp from a derived $m d r 1$-transfected cell line. In the present work, the consequences of P-gp transfers in MCF-7 were studied by using, as a P-gp donor, a cell line variant obtained by selection for resistance to doxorubicine (Doxo) and therefore called MCF-7/Doxo. MCF-7/Doxo have previously been shown to overexpress P-gp, to be resistant to doxorubicin and to efflux $\mathrm{P}$-gp-substrate fluorescent probes in a verapamil and cyclosporine A-sensitive manner [28].

Resistance phenotypes were first assessed both in MCF-7 and MCF-7/Doxo by determining growth rates in the presence of increasing Doxo concentrations $(0$, $0.1,0.3,1,3$ and $10 \mu \mathrm{M})$. As seen in Figure 1, without treatment, MCF-7 and MCF-7/Doxo displayed very similar growth rates, 0.6195 and 0.6328 per day, respectively. These values correspond to a doubling time of 26.8 and 26.3 hours, for sensitive and resistant cells, respectively. In the presence of Doxo (0.1-3 $\mu \mathrm{M})$, the proliferation rates of MCF-7/Doxo were not significantly modified. By contrast, MCF-7 cells were strongly affected by the treatment, the growth rates passing through 0.0316 per day at $0.1 \mu \mathrm{M}$ Doxo and decreasing very rapidly to -0.7492 per day with $3 \mu \mathrm{M}$ Doxo.

\section{Transfers of P-gp and efflux activity in co-cultures}

In order to investigate potential transfers of $\mathrm{P}$-gp between cells, $50 \%$ of non P-gp-expressing parental MCF-7 cells were co-cultured with $50 \%$ of their multidrug resistant counterpart MCF-7/Doxo. Obvious morphological differences allow distinction of MCF-7 from MCF-7/Doxo variants in the monolayer under phase contrast microscopy (Figure 2). After attachment to the tissue culture treated dish, MCF-7 were characterized by markedly birefringent membrane margins, while MCF-7/Doxo were more spread with dark edges and flat cell bodies. Following several days of growing in co-culture, we noticed a singular and stable spatial organization consisting in the formation of MCF-7 islets encased in a layer of MCF-7/Doxo.

A first approach used to detect a potential intercellular P-gp transfer was direct immunodetection of P-gp in living cells in the co-cultures, by using a phycoerythrinconjugated monoclonal antibody directed against an epitope localized in an extracellular loop of the protein [29]. Membrane P-gp content was followed over time, from day 0 to day 6 , by flow cytometry. As shown in Figure 3A, the peak corresponding to non P-gp-expressing MCF-7 at day 0 progressively drifted to the right from $3.60 \pm 0.53(\mathrm{n}=10)$ to $14.53 \pm 2.34(\mathrm{n}=4$, mean S.E.M. $)$ arbitrary fluorescence units at day 6 of co-cultures, in our experimental conditions. In addition, the total mass of $\mathrm{P}$-gp remained unchanged over time for 10,000 analyzed cells (Figure $3 \mathrm{C}$ ). Thus, it is suggested that the distribution of P-gp within the cell population was reorganized with time but that the balance between P-gp biosynthesis and degradation remained constant, in average, in the samples.

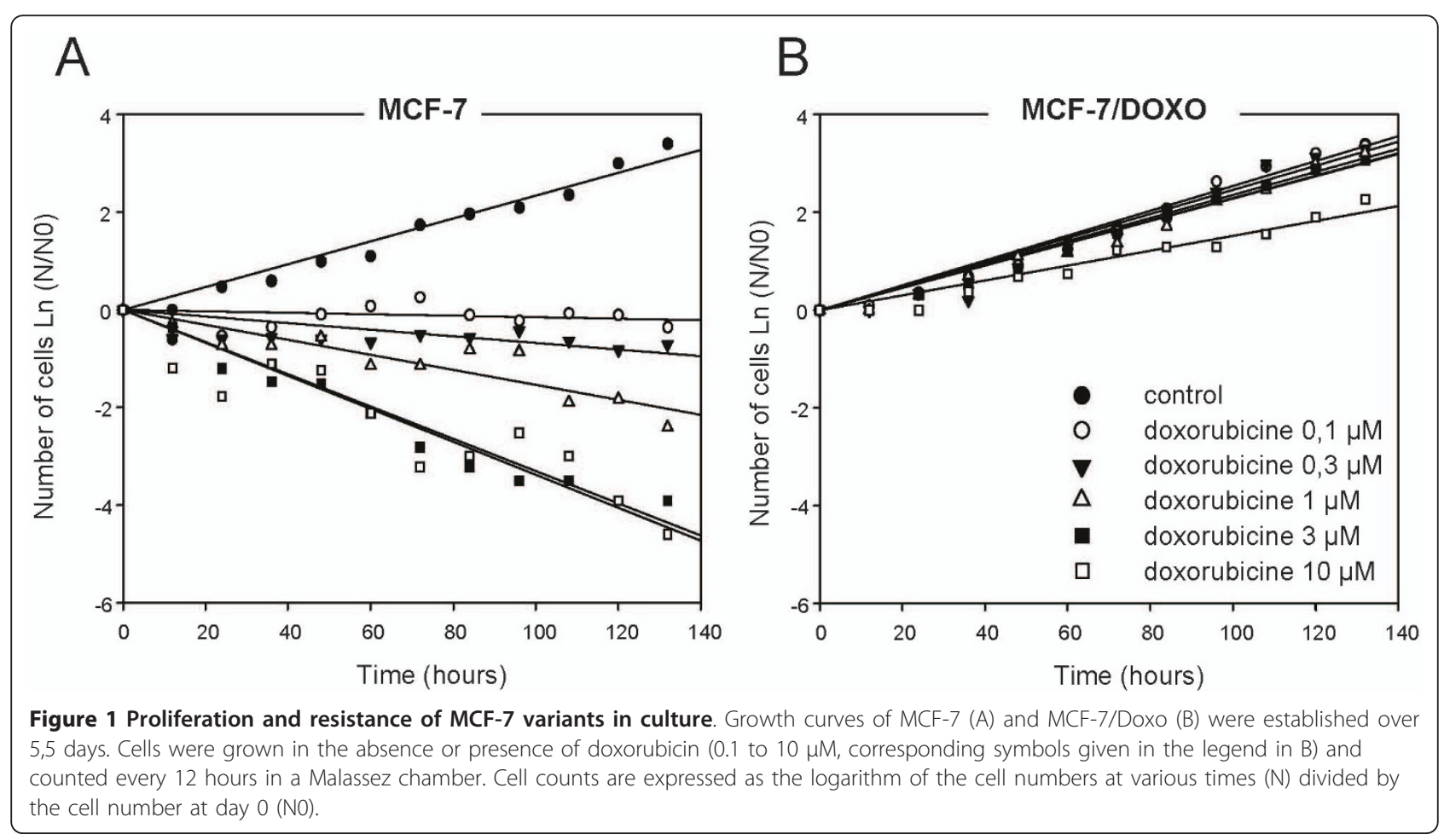




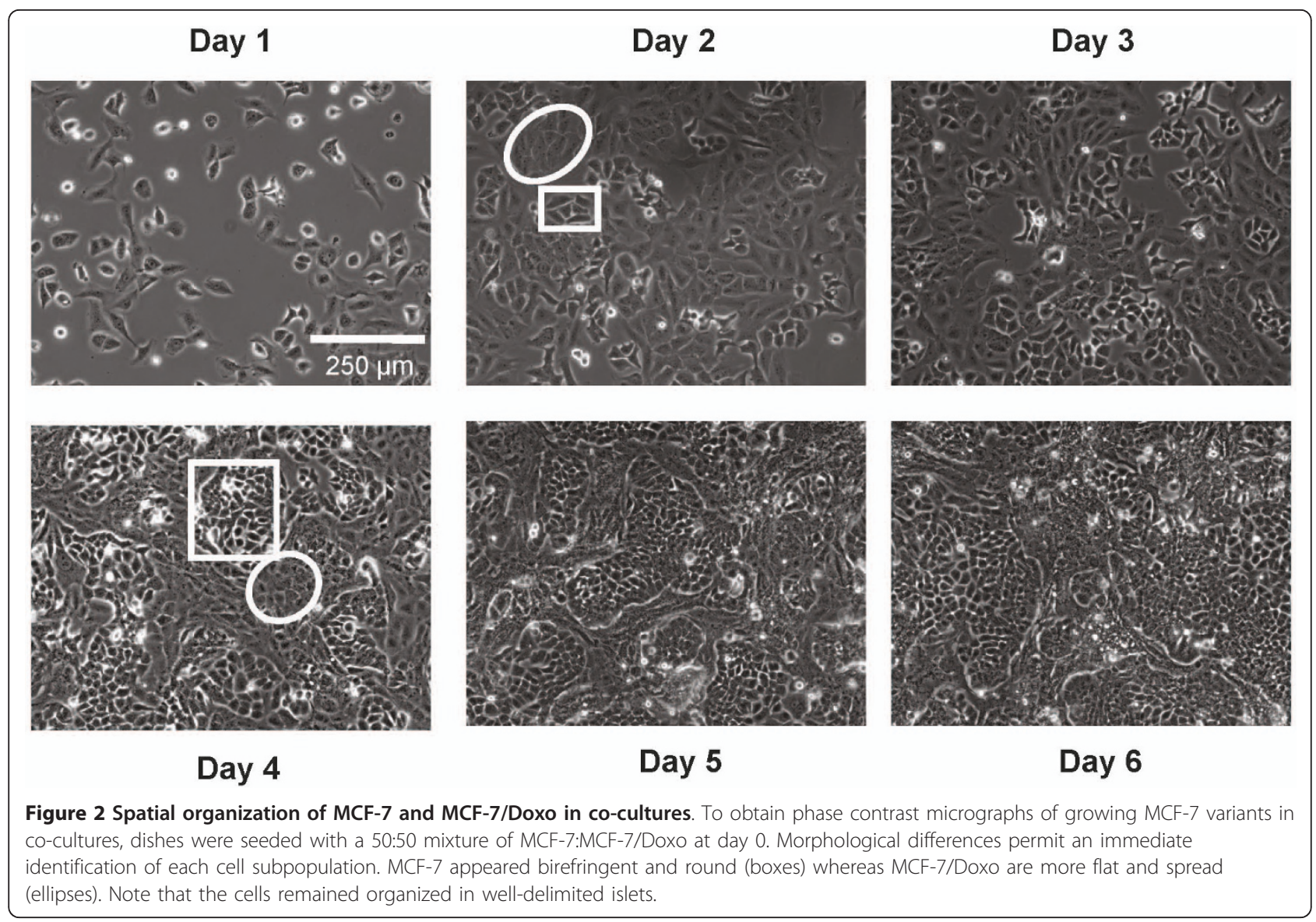

To investigate whether P-gp transfers generate a functional drug resistance, the ability of co-cultured cells to efflux calceinAM, a P-gp substrate probe which is a precursor of the fluorescent dye calcein, was assayed again by flow cytometry. In these experiments, cell fluorescence accumulation is therefore an inverse function of P-gp activity. Symmetrically to what observed with membrane P-gp content, the peak of fluorescence corresponding to initially sensitive cells, with a high calcein concentration, was also progressively shifted, but to the left, towards regions of less fluorescence accumulation (Figure 3B). The fluorescence values decreased from $2553 \pm 227(\mathrm{n}=9)$ at day 0 to $322 \pm 31(\mathrm{n}=21$, mean \pm S.E.M.) at day 6 of co-cultures. These cells therefore acquired an aptitude to efflux the fluorescent dye. However, by contrast with Figure 3A, the activity distribution is additionally characterized by the appearance of a third subpopulation displaying an intermediate efflux activity. This middle peak increased in amplitude with time to reach $32.5 \pm 1.6 \%$ of the cells at day 6 of co-cultures $(n=$ 21 , mean \pm S.E.M.). Concurrently, the peak amplitudes corresponding to the subpopulations of MCF-7 with higher and, especially, lower calcein concentrations slightly declined. Interestingly, the total mass of activity (Figure 3D) also remained constant with time.

Although the progressive shift towards regions of higher membrane P-gp content, concurrently with the appearance of higher efflux activities, are observations that can be accounted for by effective P-gp transfer in co-cultures, the exact origin of these protein and activity redistributions had to be clarified. It should be first mentioned that, in our experiments, such phenomena are never observed in cells cultured separately. Nevertheless, in co-cultures, various cell interactions as well as secretion of soluble factors, could be responsible for microenvironmental regulations resulting in a decrease in P-gp expression in MCF-7/Doxo. Such phenomena could result in $\mathrm{P}$-gp and activity redistributions, consistent with results observed in the preceding experiments. To investigate these points, it was necessary to distinguish and to follow both MCF-7 variants in co-cultures. Thus, parental sensitive MCF-7 cells were tagged with the persistent dye Cell Tracker Blue (ctbMCF-7) before mixture with MCF-7/Doxo and co-culture. As seen in Figure 4, upon one day of co-culture, a population of ctbMCF-7 appeared also positive to $\mathrm{P}$-gp detection with 


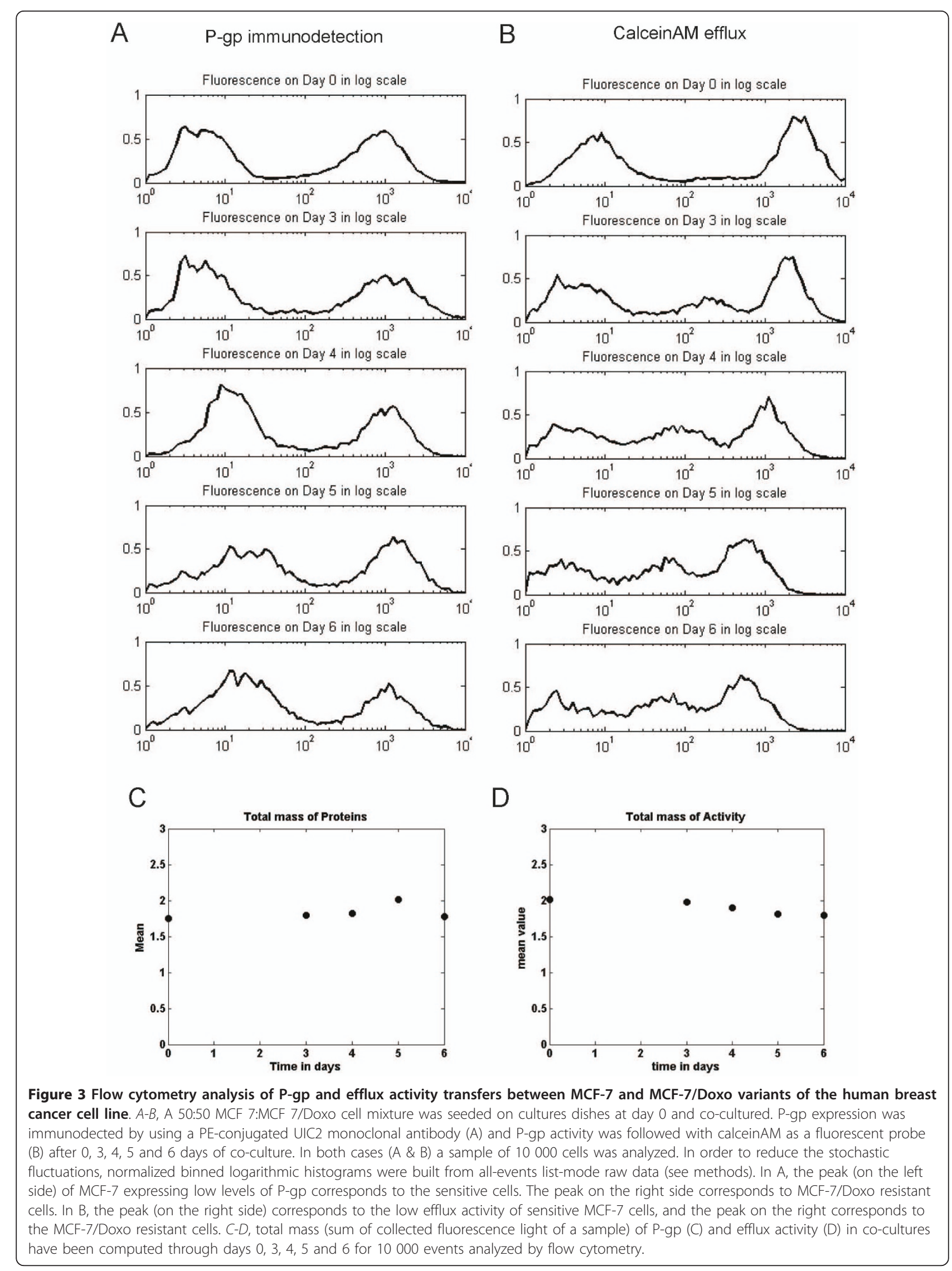

B CalceinAM efflux 
the phycoerythrin-conjugated UIC2 mAb. This population appeared to be relatively stable over 3 days of coculture, even though dilution of Celltracker blue within successive ctbMCF-7 generations weakened the signal and did not allow us to distinguish cell subpopulations further. In these experiments we also noticed continuous variation of phycoerythrin fluorescence in co-cultured ctbMCF-7, suggesting a progressive acquisition of P-gp by sensitive parental MCF-7, rather than a decrease of P-gp expression in MCF-7/Doxo. The possibility that dual positive events were, in fact, artifactual doublets of ctbMCF-7+MCF-7/Doxo can be excluded because the cytometry analysis was triggered on the electronic Coulter-type cell volume (EV) channel. Cell diameters in quadrants Q1, Q2 and Q4 matched to values corresponding to individual MCF-7 or MCF-7/ Doxo cells (Figure 5).

Taken together, these results strongly suggest the occurrence of a mechanism of transfer $i$ ) involving only a small fraction of the membrane content in protein of the donor cells but conferring a significant efflux activity to the recipient cells and ii) exhibiting a continuous exchange mode simply dependent on the gradient, i.e. the net difference in P-gp expression levels separating the cells under concern. In order to test these hypotheses and to evaluate their consequences in the dynamics of multidrug resistance within a cell culture, a mathematical transfer model was developed.

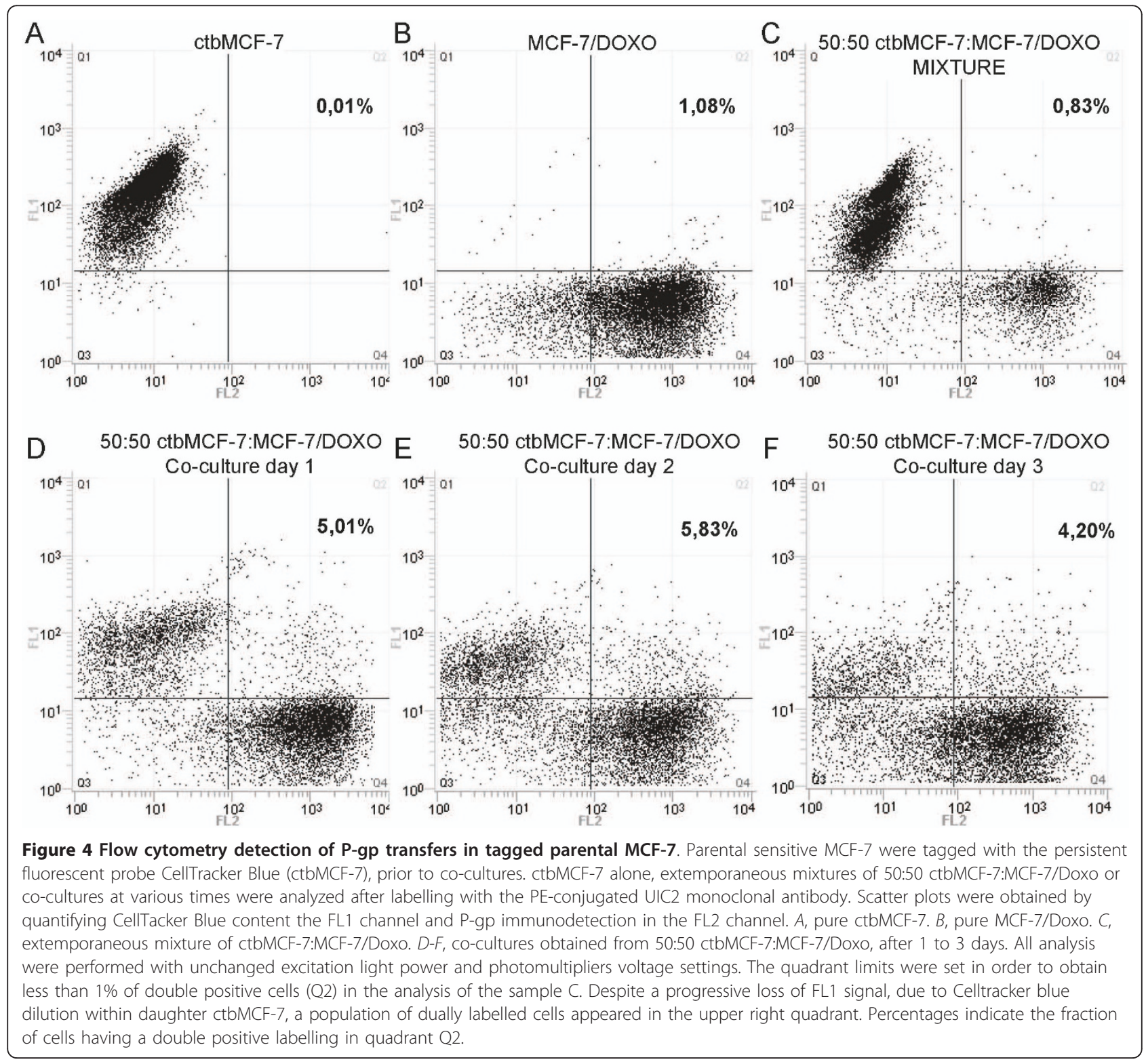




\section{Estimation of transfer parameters}

To quantify the transfer of P-gp activity, which is assumed to result in a gain of resistance, we have used a mathematical model, presented in the material and methods section and derived from Hinow et al. (2009). In the absence of cytotoxics and according to Figure 1, the growth rate is independent of the P-gp content. Herein, the growth rate was thus assumed to be identical for MCF-7 and MCF-7/Doxo in co-culture ( $\rho=0.63$ day $^{-1}$ ).

The transfer rate $\tau$, the transfer efficiency $\mathrm{f}$, and the thresholds $\delta_{\min }<\delta_{\max }$ were estimated by using intensive parameter estimation computations. The conditioned flow cytometry activity distribution (see material and methods) at day 0 was used as an input for the numerical simulation of the model.

The best fit to data distribution at day 6 was estimated by using the least square minimization method over 100,000 different values of the parameters (Figure 6). An algorithm was implemented to search for the minimal Euclidian distance between the experimental data and the numerical simulations at day 6. More precisely, we used the experimental data to define the initial distribution and to compute the solution of theoretical model. Then, we looked for the parameters providing the minimal Euclidian distance between the theoretical distribution and the experimental one at day 6 .

Conclusively, the model is coherent with the existence of cell-to-cell resistance transfers, since the best fit of the parameters gives $\tau=0.4$, and $\mathrm{f}=0.2$, corresponding to non-null transfer rate and efficiency. The parameters of the model, the respective methods used to determine their numerical values and the associated symbols are given in Table 1.

Fitted parameters seem to indicate that some limitations, or constraints, occurred for transfers between cells in our experimental conditions. First, the transfers were not homogeneous over the whole assortment of activities expressed by cells within the co-cultures. In this respect, a threshold $\delta_{\text {min }}$ distinct from 0 suggests that transfers did not occur or were not detected when $\mathrm{P}$-gp activities of the cells were to close. In addition, since the threshold $\delta_{\max }$ is not equal to the maximal value $10^{4}$, transfers did not arise either in case of extreme activity differences. This implies the occurrence of a permissible range of activities governing the optimal transfers between donor and recipient cells. Moreover, a transfer rate of $\tau=0.4$ means that transfer events occurred in average every $1 / \tau=2.5$ days for each cell in our experiments. Finally, the quantity of transferred activity globally corresponds to a fraction $\mathrm{f}=0.2$ of the difference between the activity of the donor and recipient cell.

\section{Consequences of resistance activity transfers on model response to chemotherapeutic cycles in co-cultures}

Fast acquisition of P-gp activity by doxorubicin-sensitive MCF-7 in a population of growing tumoural cells may be of first relevance to determine the dynamics of response to chemotherapy. Thus, to test the significance of P-gp transfers within a co-culture of sensitive and resistant cells, simulations were carried out by using our mathematical model of activity transfers, fed with biological data. 


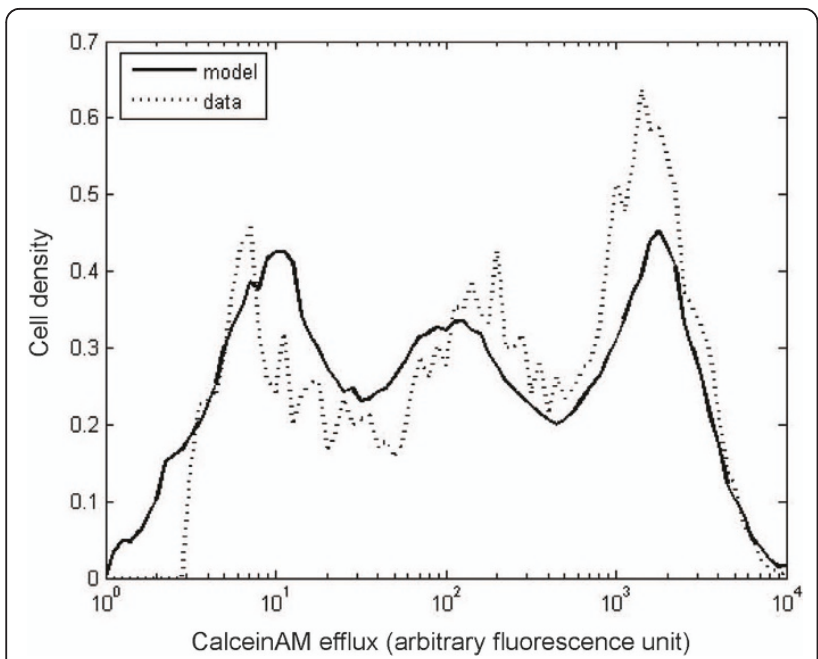

Figure 6 Estimation of parameters for the transfer model. This graph corresponds to the distribution of P-gp activity at day 6 of co-culture. The initial distribution of P-gp activity at day 0 (not shown here) was obtained from a mixture of 50:50 MCF-7:MCF-7/ Doxo cells analyzed by cytometry as in Figure 4 . The transfer model was run over 6 days. The dotted curve corresponds to the efflux activity of the co-culture measured at day 6 , and the solid curve corresponds to the distribution of activity derived from model. The fitting parameters are given above the curves and were obtained by least squares minimization.

Herein, the simulations were built with basic parameters determined in vitro, in particular the growth rates of monolayers and the transfer parameters. Therefore, cell proliferation was artificially high in abstraction of any physiological environment and the growth curves obtained by simulation should not be considered as data clinically exploitable. Likewise, practical aspects of treatment administration involving pharmacokinetics of doxorubicin (such as elimination half-life, drug clearance, volumes of distribution) were ignored. The simulated treatment was simplified into a bolus of $1 \mu \mathrm{M}$ doxorubicin that reaches the maximum concentration in the extracellular compartment with no delay and maintains the same level over 10 days. Then, the treatment was interrupted for 4 days (i.e. doxorubicin concentration is zero). The treatment cycled every 14 days for 4 cycles.
As shown in Figure 7A, the number of pure sensitive MCF-7 in a simulated culture declined progressively in the presence of Doxo during the first 10 days of a treatment cycle and, thereafter, increased during the 4-days interruption at rates $\rho\left(p_{\min }\right)$ corresponding to the values previously determined (Figure 1). To follow the respective populations of sensitive and resistant cells within the simulated co-cultures, a particular attention should be paid to the criterion used to define what exactly a sensitive cell is (resistant cells being considered as the complement). Herein, we defined sensitive cells as MCF-7 having a negative growth rate in the presence of $1 \mu \mathrm{M}$ doxorubicin. The growth rate $\rho$ is supposed to vary only as a function of the overall $\mathrm{P}$-gp activity $p$ (intrinsic activity and activity acquired or lost by transfers), as presented in supporting Figure 7B. As a consequence, transferred sensitive cells with enough P-gp activity to have a positive growth rate will be considered as resistant cells.

The evolution of the simulated co-cultures was extremely dependent on the initial distribution of cells, i.e. the relative proportions of cells of with different P-gp contents. Again, as for the estimation of transfer parameters, the biological data distribution in term of efflux activity $p$ at day 0 , was used as an input template for computer simulations. However, the simulations were started with inocula of $10^{4}$ cells (corresponding to an approximate mass of $0,01 \mathrm{mg}$ ) comprising only a small proportion of resistant cells, 0.1\% (i.e. 10 cells, Figures $8 \mathrm{~A} \& 8 \mathrm{~B}$ ), or $1 \%$ (i.e. 100 cells, Figures $8 \mathrm{C} \& 8 \mathrm{D}$ ). For each condition, the numbers of sensitive, resistant and total cells were plotted as a function of time in two situations: without or with cell-to-cell P-gp activity transfers.

In the absence of transfer (Figure 8A\&8C), resistant cells grew linearly, and represented after few days the majority of the cells in the co-cultures. The number of sensitive cells decreased during the treatment and increased during the interruption phases. In these simulations, the initial distribution of sensitive cells was based on low efflux activities analyzed by flow cytometry. As a consequence, the population of sensitive cells did not correspond to a single ray but was rather represented by a bell curve shape encompassing a spectrum

Table 1 List of the model parameters, their significations, values and symbols

\begin{tabular}{llll}
\hline Symbol & Interpretation & Value & Units \\
\hline$\rho$ & Growth rate of cells in absence of drug & 0.63 & Method \\
$c$ & Drug concentration & 0 or 1 & $\mu M$ \\
$\tau$ & Rate of transfer of P-gp activity & 0.4 & day $^{-1}$ \\
$F$ & Activity transfer efficiency & 0.2 & - \\
$\delta_{\min }$ & Minimum threshold for transfers & $10^{1.4}$ & fluorescence unit \\
$\delta_{\max }$ & Maximum threshold for transfers & $10^{1.9}$ & fluorescence unit \\
\hline
\end{tabular}

The column method refers to the approach employed to determine their respective numerical value. 


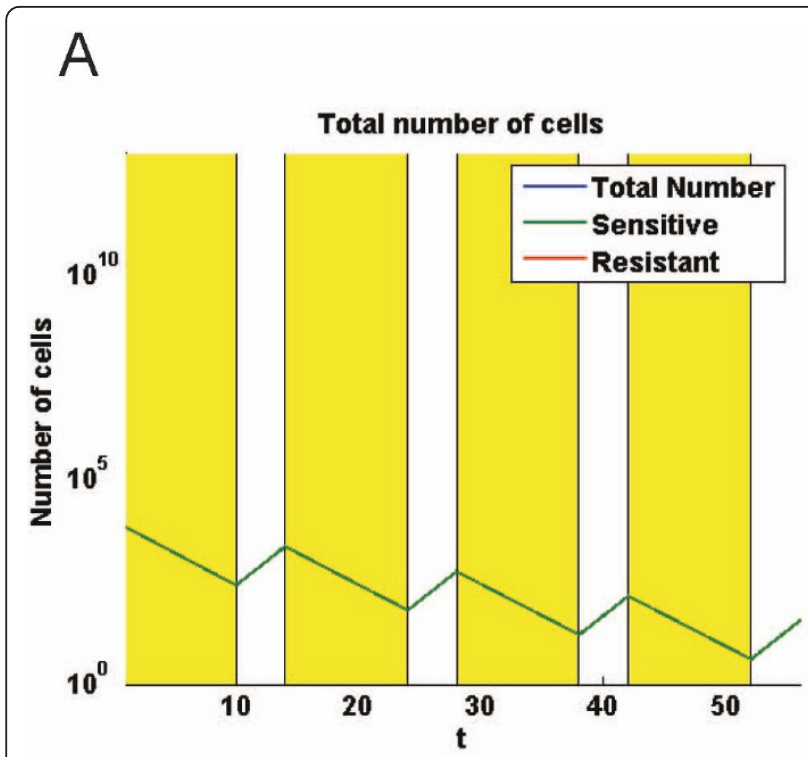

B

Figure 7 Growth rate of sensitive cell as a function of P-gp content. $A$, computer simulation giving the number of sensitive MCF-7 versus time during a chemotherapy regimen, cycling every 14 days, with 10 days of $1 \mu \mathrm{M}$ doxorubicin treatment followed by 4 days of interruption, for 4 cycles. The initial simulated tumour consisted in $10^{4}$ MCF-7 having growth rates corresponding to $\rho$ ( $p_{\text {min }}$ ). $B$, plot giving the growth rates $\rho$ as a function of the P-gp-driven calceinAM efflux activity $p$ for cells exposed to $1 \mu \mathrm{M}$ doxorubicin. The function describing $\rho(p)$ is used in the simulations presented Figure 8. All cells having a negative growth rate are considered as sensitive MCF-7.

of efflux activities, from zero to several arbitrary fluorescent units. Consequently, from a cycle to another, the sensitive cells having the less negative growth rates were selected by the treatment. For that reason, the population of sensitive cells decreased to a minimum and, then, increased again by growing during treatment interruptions while the growth rates converged to zero during the doxorubicin phases.

In the presence of transfers (Figure 8B\&8D), results differed from above and were greatly influenced by the initial number of resistant cells in the inoculum. In fact, with transfers during treatment, growth rates of resistant and sensitive cells converged to an intermediate value, due to a loss of activity by resistant combined with a gain of activity by sensitive cells. With initial conditions corresponding to $0.1 \%$ or $1 \%$ of resistant cells, after an initial phase during the first treatment, the growth rates of the cell population tend to become analogous. The main observation is that transfers significantly decreased the growth rate of the whole cell population exposed to cytotoxics, especially when the co-culture is initiated with a relative large proportion (1\%) of resistant cells.

\section{Discussion}

In the present work, we observe effective transfers of $\mathrm{P}$-gp and efflux activity from a P-gp overexpressing MCF-7 human breast cancer cell line variant, selected for its resistance towards doxorubicin, to the parental sensitive cell line. Our results thus confirm the work of

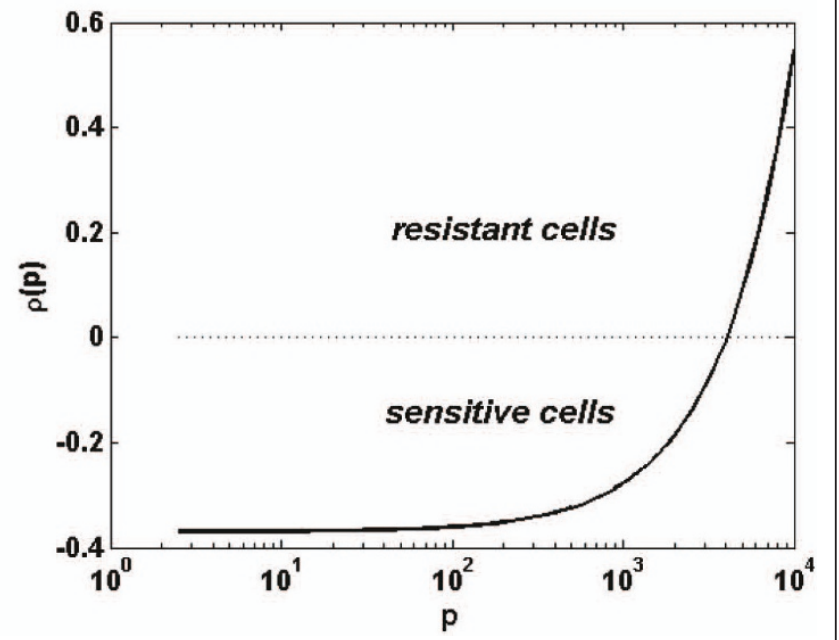

Levchenko et al. (2005) conducted in vivo and also in vitro on several adherent cell lines, including MDR1transfected MCF-7 [24]. Functional transfers of P-gp have also been described very recently between leukemic cells cultured in suspension [25]. In addition, the possibility for a malignant cell of inheriting P-gp from a normal cell was shown by Rafii et al. in a study where sensitive ovarian cancer cells acquired P-gp by interaction with particular stromal cells isolated from the peritoneal cavity of patients [24]. To our best knowledge, the present work and the three above cited references are the first reports describing extragenetic direct cellto-cell transfers of P-gp. Among these lines, it should be mentioned that an intercellular transfer of the drug resistance phenotype was first reported indirectly in a pioneering study of Frankfurt et al. [30].

The present data highly reinforce the fact that the P-glycoprotein, a $170 \mathrm{kDa}$ polypeptide consisting of 1280 amino acids that spans 12 times the cell membrane [31], can be exchanged from a donor cell to a recipient one, keeping its ability to efflux drugs and, thus, conferring the MDR phenotype. In addition to the discovery of the ability, for cells, to transfer huge integral membrane proteins, these important findings also reveal the occurrence of an additional extragenetic mechanism to acquired chemoresistance, in the absence of pressure selection, in vitro. The possible occurrence of such P-gp transfers in vivo may be crucial to understand P-gp expression in breast cancers. In this context, 


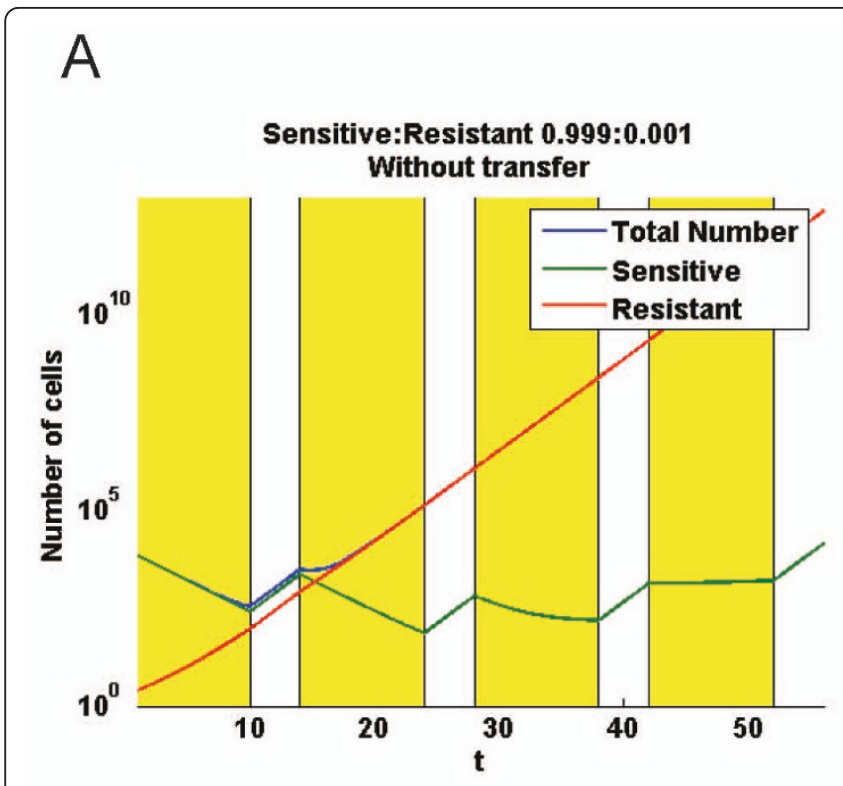

C

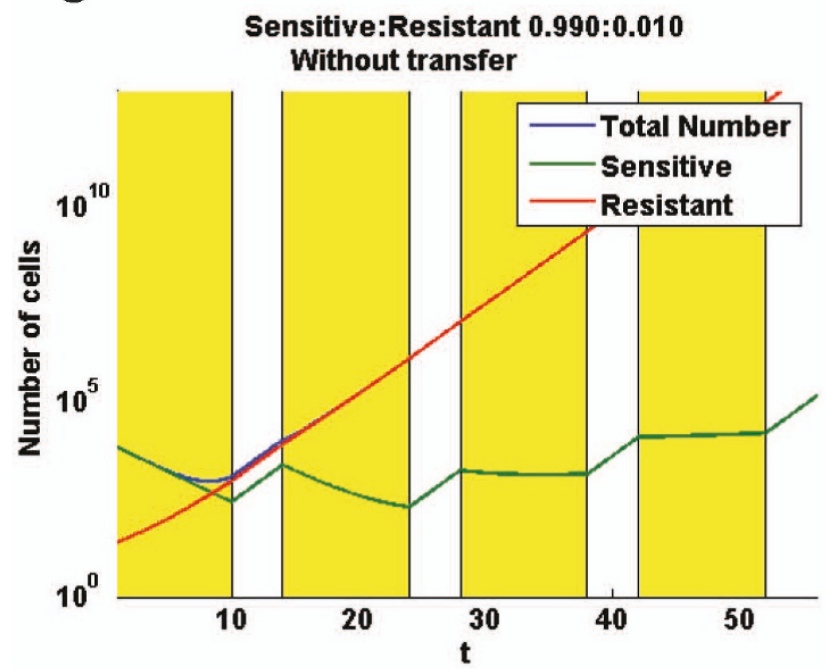

B

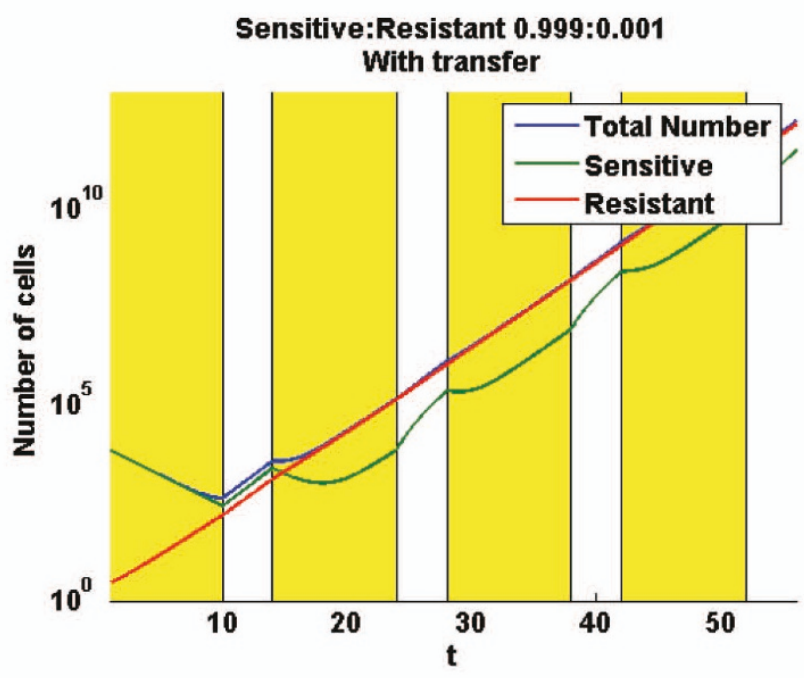

D

Sensitive:Resistant 0.990:0.010 With transfer

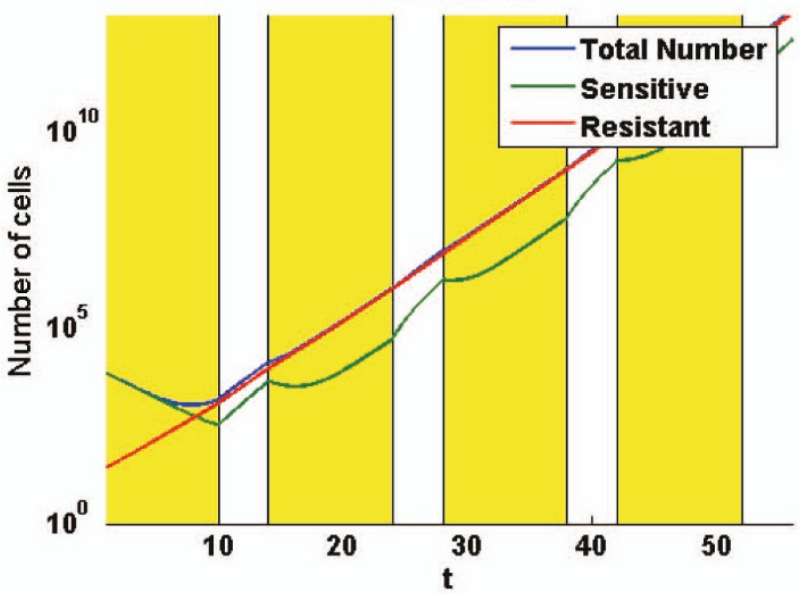

Figure 8 Computer simulation giving the consequences of cell-to-cell P-gp activity transfers in co-cultures exposed to doxorubicin, according to the mathematical model. A chemotherapy regimen is simulated by cyclic exposure of an inoculum of $10^{4}$ cells to $1 \mu \mathrm{M}$ doxorubicin. The treatment cycled every 14 days, with 10 days of doxorubicin treatment followed by 4 days of interruption, for 4 cycles. The initial proportions of resistant MCF-7/Doxo vary form $0,1 \%(A \& B)$ to $1 \%(C \& B)$. Simulations were performed in situations where transfers were abolished $(A \& C)$ or permitted $(B \& D)$ with the parameters determined in Figure 6 . For each condition, the numbers of sensitive, resistant and total cells are plotted versus time. Sensitive MCF-7 are defined as cells having negative growth rate in the presence of $1 \mu$ M doxorubicin (see Figure 7).

it should be remarked that data concerning P-gp expression in human breast tumours are known to suffer of considerable variability across studies, as revealed by a compendium based on 31 articles compiling results obtained mainly by immunodetection methods onto 1,200 breast carcinomas [13]. The discrepancies in the literature were ascribed in a large extent to a lack of rigorous methodology, preparation of samples as well as
anti-P-gp antibodies varying from a study to another $[32,33]$. Some immunohistological studies carried out in normal breast collected from healthy donors failed to find P-gp expression $[34,35]$. However, by using a more sensitive approach, namely RT-PCR research for P-gp transcripts, low but significant levels of P-gp mRNAs have been detected in normal tissues adjacent to chemotherapy-naïve tumours [36]. Importantly, low basal 
levels of P-gp efflux activities have been shown to be sufficient enough to contribute extensively to drug resistance, in vitro [37]. In this context, the levels of P-gp activities heritable by P-gp transfers and found in the present study are largely compatible with a resistance increase in vivo. The time-course of P-gp transfers, detectable between the third and the fourth day in coculture, is also short enough to overcome the effects of cytotoxics used in chemotherapy.

The particular spatial organization of MCF-7 in cocultures has certainly severe consequences on the contact surfaces between sensitive and resistant cells. As seen under microscopic observation, cells were not organized as a homogeneous mixture of well-separated cell variants, but rather in delimited islets of sensitive settling aside from resistant cells. As a result, transfers between the most separated populations in term of chemoresistance (full sensitive versus full resistant cells) may occur only at the frontier of islets in case of cellto-cell contact-dependent transfers [23,24], keeping in mind that transfers can also arise between cells of intermediate P-gp levels. From this, it is assumed that the transfer process is a rather complicated phenomenon in which spatial structures bring limitations. Such spatialconstraint or regulated P-gp transfers may provide an additional rationale to the heterogeneity of P-gp expression previously observed in studies carried out on heterogeneous tumour samples [13,33]. Additional studies focused on mechanisms of P-gp transfers are needed to go further concerning that point.

As a matter of fact, our approach to quantify the parameters of transfers by using biomathematical modelling, points out some constraints, revealed by the parameters fitted to the biological data. The mathematical model has been constructed to $i$ ) be applicable to a population of interacting cells in proliferation, $i$ ) to consider the cell population as a continuum density structured by the quantity $p$ of transferable P-gp activity and iii) to have rules governing transfer. The rules of the transfer process have been delineated and discussed in detail in a companion paper [26]. The better fit was obtained by using the activity transferred from resistant to sensitive cells, undirected and directed shifts being neglected. Herein, we consider that P-gp activity is a better estimation of therapy resistance as compared to P-gp expression, because activity is directly responsible for resistance to cytotoxic drugs. Moreover, this choice allows us to use directly the model to simulate the response of a co-culture to chemotherapeutic cycles (see below). Nevertheless, a correlation between P-gp levels and activity has been described in several cell lines $[15,38,39]$. Parameters fitting revealed quantitative aspects of $\mathrm{P}$-gp activity transfer, in particular a transfer rate $\tau$ corresponding to one event every 2.5 days, a value to be compared to the doubling time of MCF-7 close to 1 day. In addition, the calculation of the average activity and the average level of P-gp expression over the time in a co-culture showed that theses values remain stable throughout the experiments. We conclude that there is neither significant gain nor loss of efflux activity and Pgp expression during the co-culture experiments. As a consequence, the changes in the fluorescence distributions (for both P-gp and activity) can be attributed to transfers, which are not rare phenomena compared to the cell cycle duration. However, in a culture of growing cells transferring P-gp, the topology of the exchange network is constantly changing. This aspect is not taken into consideration in the transfer model which does not adapt rules of transfer as a function of time. The estimation of the transfer rate $\tau$ is therefore a coarse estimation that should be re-evaluated in future works including spatial dynamics.

The model gives also a transfer efficiency $f=20 \%$, corresponding to the amount of activity transferred each time with regard to the absolute activity difference between the donor and recipient cells, and two transfer thresholds, that can be viewed as bottom and top activities delimiting authorized transfers. These parameters can be related to the life span of potential transfer vectors, that are constructed and destroyed for instance, or to the size of single P-gp cargos. Concerning this point, it has been shown that high density P-gp microdomains alter local properties of lipid membrane environment and promote P-gp clustering [40]. In this respect, biological data as well as transfer parameter $f$ suggest an organized quantal transfer of P-gp, in which the minimum unit of exchange would probably consist in a cluster of several copies of the transporter. Several intercellular carriers have been proposed to be involved in P-gp transfers. In adherent cells lines, close contacts between donor and recipient cells have been shown to be required $[23,24]$. In the other, in a model of liquid tumour, P-gp containing microparticles released by resistant cells and bound to sensitive cells, have been isolated [25]. The possibility that both mechanisms actually co-exist in our cell line is examined in an independent study [27].

The model was used to simulate and to quantify the consequences of P-gp activity transfers on the cell distribution within a growing co-culture during exposure cycles to cytotoxics. More precisely, the model was fed with biological data corresponding to sensitivity of MCF-7 variants to doxorubicin. Doxorubicin is among the preferred agents used in chemotherapy regimens for preoperative breast tumour reduction, adjuvant treatment after a first line surgical lumpectomy/mastectomy and radiotherapy of invasive breast carcinoma or systemic treatment of recurrent or metastatic breast cancers [41]. Doxorubicin is administrated alone or in 
combinations with other cytotoxics. In the present simulations, the concentration of doxorubicin was set at $1 \mu \mathrm{M}$, a value corresponding to the weekly supplementation of MCF-7/Doxo culture medium. This concentration is also close to the levels of doxorubicin found in tumours under chemotherapy, reported to vary in the $10-0,1 \mu \mathrm{M}$ range depending on the time separating the administration of the drug to the measures [42-44].

The major consequence of P-gp activity transfers is that subpopulations of sensitive and resistant cells were no longer compartmentalized. Via P-gp activity transfers, flows of cells depopulate and repopulate resistant and sensitive cells. This was obvious once the second cycle of treatment began, where the population of sensitive cells increased by adjunction of cells coming from resistant cells, which lose P-gp activity. Conversely, sensitive cells acquired $\mathrm{P}$-gp activity and, thus, developed progressively positive growth rates during the treatment phases. When the number of donor cells was increased up to $1 \%$, the resulting exchanges of $\mathrm{P}$ gp caused a significant loss of activity for the resistant cells. Acquisition of P-gp activity was therefore slowed down for sensitive cells. As a result, in that case, the numbers of sensitive and resistant cells within the coculture converged (became close) with time and the overall growth rate of the cell population was reduced (compared to all the other situations), giving a co-culture with fewer cells at the end of the four chemotherapeutic cycles. In our model, the gain of $\mathrm{P}$-gp is central for survival and growth of non P-gp expressing cells whereas the loss of P-gp by resistant cells has predominant effects on the overall growth rate of the simulated tumour. We also conclude that the initial proportion of resistant cells in a population has a great influence on the growth of the whole population in the presence of transfer.

The present study sheds new light on fundamental features underlying extragenetic acquisition of multidrug resistance in vitro, with a particular attention given to quantitative aspects. Globally, P-gp transfers led to an integration of the responses to the drug across the cell population and a 'collective' phenotype different than the sum of parts. However, MCF-7/ Doxo represents an extreme case of P-gp overexpression and fast growing cells. Future work should be conducted with cells expressing intermediate amounts of efflux activity, since transfers have been demonstrated to be dependent on the P-gp levels [23]. Moreover, an improved mathematical model taking into account evolving 2D or 3D spatial considerations has to be developed together. Physiopathological implications of cell-to-cell protein transfers may be crucial, especially if other membrane proteins and various cell types are concerned.

\section{Material and Methods \\ Cell lines}

The cell lines used in the present study were the wild-type drug-sensitive human breast adenocarcinoma MCF-7, purchased at the American Type Culture Collection (LGC standards, Molsheim, France), and a multi-drug resistant MCF7/Doxo variant, kindly provided by Pr. J.-P. Marie (Hôtel Dieu, Paris, France). The cells were grown in RPMI 1640 medium supplemented with $5 \%$ heat-inactivated fetal bovine serum, $2 \mathrm{mM}$ L-glutamine, and 1\% antibiotic/antimycotic solution. Doxorubicin $(1 \mu \mathrm{M})$ was added to the culture medium for the maintenance of the multi-drug resistant phenotype of MCF-7/doxo cells. Cultured cells were incubated at $37^{\circ} \mathrm{C}$ under a water-saturated $95 \%$ air-5\% $\mathrm{CO}_{2}$ atmosphere. Exposure of MCF-7/Doxo to the P-gp inhibitors verapamil or cyclosporine A dose-dependently abolished both resistance to doxorubicin and P-gp activity in MCF-7/doxo [28].

For proliferation assays, cells were plated at $10^{5}$ per $60 \mathrm{X}$ $15 \mathrm{~mm}$ tissue culture dish with or without doxorubicin. Cells were dissociated by treatment with trypsin/EDTA and then counted in a Malassez chamber. Cell proliferation was followed every 12 hours during six days.

\section{Analysis of P-gp expression and activity by flow cytometry}

Cultured or co-cultured MCF-7 were trypsin-resuspended and washed with HBSS before analysis. For intercellular P-glycoprotein transfer studies, P-gp was labelled using a phycoerythrin (PE)-conjugated UIC2 mAb (Beckman Coulter France, Villepinte, France). The fluorescent light (FL) was quantified using a Cell Lab Quanta SC MPL flow cytometer (Beckman Coulter) equipped with a $22 \mathrm{~mW}$ $488 \mathrm{~nm}$ excitation laser. Voltage settings of photomultipliers were not modified throughout the experiments. Each analysis consisted in a record of 10000 events, triggered on electronic volume (EV) as primary parameter, according to a particle diameter exceeding $8 \mu \mathrm{m}$. Red fluorescence was measured in FL2 channel (log scale) through a $575 \mathrm{~nm}$ band pass emission filter. More than $93.1 \pm 0.4 \%$ (mean \pm standard error of the mean) of gated events exhibited a FL2 > 1 for MCF-7/Doxo.

To study P-gp activity, resuspended cells were loaded with $0.25 \mu \mathrm{M}$ calcein acetoxy-methylester (Invitrogen Life Technologies, Carlsbad, CA) in RPMI for 15 min at $37^{\circ} \mathrm{C}$ in the dark. Green FL was quantified via the FL1 channel (log scale) through a $525 \mathrm{~nm}$ band pas filter. Controls of MCF-7, MCF-7/Doxo and extemporaneous mixtures of 50:50 MCF-7: MCF-7/doxo were analysed before co-cultures, in all experiments.

\section{Analysis of P-gp by flow cytometry transfer in tagged MCF-7}

75\%-confluent sensitive MCF-7 were loaded with $25 \mu \mathrm{M}$ of the cell-permeant reactive fluorescent dye CellTracker 
Blue 4-chloromethyl-6,8-disulforo-7-hydroxucoumarin (Invitrogen Life Technologies, Carlsbad, CA) for 2 hour at $37^{\circ} \mathrm{C}$ in $25 \mathrm{~cm}^{2}$ culture dishes. The cells are then washed twice with HBSS and covered with fresh complete culture medium. Either CellTracker Blue loaded sensitive MCF-7 (ctbMCF-7), or MCF-7/doxo, or a carefully homogenized 50:50 mixture of ctbMCF-7:MCF-7/ doxo were plated at the desired density on $60 \mathrm{~mm}$ culture dishes and analysed by flow cytometry after labelling using the PE-conjugated UIC2 mAb, as described before.

\section{Flow cytometry data conditioning and normalization}

Accurate fitting of the mathematical model to flow cytometry all-events histograms required raw data noise reduction. Thus, list-mode data were segregated into binned logarithmic histograms, with a bin width of 0.05 in log scale of arbitrary fluorescence units. The distributions were normalized, by scaling to an integral area of 1.0 , in order to obtain a density of probability.

\section{Mathematical model of P-gp activity transfer}

In this section, we describe a mathematical model that has been developed and used to describe the consequences of a cell-to-cell $\mathrm{P}$-gp protein transfer in terms of multidrug resistance activity. Herein, the resistance activity is viewed as the ability of a cell to efflux cytotoxics, which is considered to be correlated to the number of P-gp copies within the cell membrane.

Our modeling approach views the transfer of P-gp proteins between cells as a stochastic process in which individual transfer events are represented in a continuum population density function $u(t, p)$, where $t$ is time and $p$ is the quantity of $\mathrm{P}$-gp on individual cell membranes. The density function $u(t, p)$ is the solution of an integro-partial differential equation of Boltzmann type, which describes an evolving population behavior as an average of individual transfer events governed by specified rules. The aggregate transfer behavior is modeled as an integral operator, with a kernel specified by the transfer rules, acting on the continuum density. We assume individual transfer events arise randomly (Rule A1), occur at a specified average rate (Rule A2), and are delimited by parameters specific to allowable quantities of P-gp transferred between paired cells (Rules A3 and A4) (see below). The large number of cells and proteins involved justifies our assumption that a continuum population density function describes the dynamic transfer processes.

Rules for the transfer model:

- (A1) The probability that a pair of individuals is involved in a transfer event is independent of their $\mathrm{P}$-gp values, and the pairing is chosen randomly from all individuals.
- (A2) The time between two transfer events follows an exponential law with mean $\tau-1>0$ (alternatively, $\tau$ is the rate of transfer per unit time).

- (A3) Let $0<f<1$ (we call $f$ the transfer efficiency). A transfer between two individuals, one with value P-gp1 and the other with value P-gp2, results in a transfer of the fraction $\mathrm{f}$ of their difference $\mathrm{f} \mid \mathrm{P}-\mathrm{gp} 1$ - P-gp2| gained by the smaller and lost by the larger of the two. - (A4) An exchange takes place only if the difference of the transfer pair P-gp1 and P-gp2 in assumption (A3) is $>\delta$ (we call $\delta \in(0,1)$ the transfer threshold).

We decompose the construction of the model by considering separately the process of $i$ ) cell proliferation and death, ii) P-gp induction and degradation rate, both, at the cell and the population level, and iii) P-gp transfer between cells. We consider $p$ the P-gp efflux activity of a cell, functionally measured by the loss of calcein fluorescence for a resistant cell compared to a sensitive one, and we introduce $u(t, p)$ the density of cells at time $t$ having a P-gp activity $p$. In the sequel, for a given cell, the fluorescence $p$ is assumed to be a function of P-gp expression on the cell surface. This function is strictly increasing.

The terminology "density of cells with respect to $p$ " means that, if $p_{1}<p_{2}$ are two quantities of P-gp activity, then the number of cells with a P-gp activity in between $p_{1}<p_{2}$ at time $t$ is

$$
\int_{p_{1}}^{p_{2}} u(t, p) d p
$$

According to the fluorescence scale used in cytometry, $p$ varies between $p_{\text {min }}=1$ and $p_{\max }=10^{4}$, and the total number of cells is given by

$$
U(t)=\int_{p_{\min }}^{p_{\max }} u(t, p) d p .
$$

In order to describe the time course of the cell distribution, we introduce the following model which has already been described in [26]

$$
\begin{aligned}
& \frac{\partial u(t, p)}{\partial t}=\underbrace{\rho(p) u(t, p)}_{\text {proliferation }}+\underbrace{2 \tau(T(u(t, \cdot))-u(t, p))}_{\text {P-gp transfer }}, \quad p \in\left(1,10^{4}\right) \\
& u(0, p)=u_{0}(p) \in L_{+}^{1}\left(1,10^{4}\right) .
\end{aligned}
$$

In this model the proliferation term combines the cell division and mortality of cells, which is assumed to depend on the activity $p$ of $\mathrm{P}$-gp only during the treatment. Robin type boundary conditions are introduced to preserve the total number of individuals when only the drift process takes place (i.e. $\rho=0$ and $\tau=0$ ). The term 
describing transfer of P-gp has been presented in detail in [26]. The parameter $\tau$ describes the time between two consecutives transfers, and the transfer operator $T$ is defined by

$$
T(\phi)(p)=\frac{\int_{-\infty}^{+\infty} \bar{\phi}(p+f(\bar{p}) \bar{p}) \bar{\phi}(p-(1-f(\bar{p})) \bar{p}) d \bar{p}}{\int_{1}^{10^{4}} \phi(\bar{p}) d \bar{p}}, \varphi \in L_{+}^{1}\left(1,10^{4}\right)
$$

where $\bar{\phi}$ extends $\phi$ by 0 outside of the interval $\left(1,10^{4}\right)$. The main idea in the construction of the transfer operator $T$ is to derive the probability of a recipient partner to acquire a level $p$ of P-gp in a transfer event from all possible donor partners. To describe a transfer event we use the following rule: if a cell $C_{1}$ and a cell $\mathrm{C}_{2}$ have, respectively, a quantity $p_{1}$ and $p_{2}$ of P-gp activity before transfer, then, after transfer, $\mathrm{C}_{1}$ (respectively, $\mathrm{C}_{2}$ ) will have an activity $p_{1}-f\left(\left|p_{1}-p_{2}\right|\right)\left(p_{2}-p_{1}\right)$ (respectively, $\left.p_{2}-f\left(\left|p_{1}-p_{2}\right|\right)\left(p_{1}-p_{2}\right)\right)$. So the fraction transferred is $f\left(\left|p_{1}-p_{2}\right|\right)$, which depends on the absolute value of the difference between $p_{1}$ and $p_{2}$ (the distance between the $P$-gp activities of $C_{1}$ and $C_{2}$ ). The model is used with constraints that correspond to a admissible interval $\left(\delta_{\text {min }}, \delta_{\text {max }}\right)$ for $\left|p_{1}-p_{2}\right|$ to allow transfer. As a consequence, when $\left|p_{1}-p_{2}\right|<\delta_{\min }$ or $\left|p_{1}-p_{2}\right|>\delta_{\max }$ there is no transfer (i.e. $f\left(\left|p_{1}-p_{2}\right|\right)=0$ ) and otherwise a constant fraction is transferred (i.e. $f\left(\left|p_{1}-p_{2}\right|\right)=\sigma$ ). Therefore, the parameter $0<\sigma<1$ is called the transfer efficiency, and $0 \leq \delta_{\min }<\delta_{\max }$ are called the transfer thresholds.

In the above model, the cell distribution of P-gp is assumed to be homogeneous in space. In practice the spatial distribution of P-gp can play an important role in the transfer process. Here we neglect this aspect. This problem will be investigated elsewhere.

\section{Reviewers' Comments}

Reviewer 1: Leonid Hanin, Idaho State University, Department of Mathematics, United States of America

This is an important and well-written paper that deals with the efflux P-glycoproteins (P-gp), exchange of P-gp between cells including a mathematical model of this process, and the role of the P-gp transfer in bringing about extragenetic resistance to chemotherapeutic treatment of breast cancer.

\section{General Comments}

1. I think more biological discussion of P-gp activity and the nature of $\mathrm{P}$-gp transfer is warranted. In particular, what is the time scale for transfer processes and is the level of cellular P-gp activity heritable?
Quantitative values, relatively to arbitrary fluorescent units given by flow cytometry, are now added in the results in term of heritable cellular protein and activity, as well. This question and the point concerning the time scale for transfers are also discussed more extensively.

2. To put formation of chemotherapeutic drug resistance into a more practical perspective, it would be helpful to mention whether normal breast tissue cells display P-gp based efflux activity and whether P-gp exchange may occur between normal and malignant cells.

The one and only article showing a possibility of P-gp transfers between normal and cancer cells is the report of Rafii et al. (2008) detailed at the beginning of the discussion. The possible basal P-gp activites in normal breast and its consequences in terms of MDR are discussed $p 18$.

3. Pp. 7-8. The mathematical model is introduced too abruptly. I believe the authors should write a paragraph or two about underlying general assumptions, the "big" stochastic picture behind their model, explain the formula for the transfer operator including the role of normalization in more detail, and give stochastic interpretation of coefficient $\tau$.

A part concerning data conditioning and normalization has been added $p 7$ in Methods section. We have also modified the presentation of the model, recalling the precise assumptions for the transfer process.

4. P. 8. The paper deals with the model of P-gp transfer with $\varepsilon=\mathrm{h}=0$. Given the predominantly biological readership of the journal, I think it would be better to omit these terms in the model and refer the reader to paper [20] for a more general model accounting for diffusion and drift.

We agree with the referee, and we modified the system accordingly to the comment.

5. Paper [20] establishes the existence, under certain simplifying assumptions, of the limiting equilibrium distribution of P-gp activity. What is it in a slightly more general case of two thresholds considered in the present work (in the absence of cell proliferation)? How fast is this equilibrium reached? Is this relaxation process slower or faster than cell cycling?

The two threshold case was in fact also considered in the paper by Hinow et al. Up to now the authors did not study the speed of convergences to the equilibrium distributions (which is indeed a difficult question). In the present model the cell cycle is simplified but includes the growth rate of cells (neglecting the oscillation induced by the cell cycle). This problem will be considered in future works.

6. What are the relative effects of P-gp transfer and cell proliferation on chemotherapeutic activity of DOXO? One can compare survival of two-compartment 
tumor cell population exposed to DOXO under two scenarios: (1) no cell proliferation, just P-gp exchange and (2) cell proliferation alone assuming equilibrium distribution of P-gp activity. This would give some idea about the net effect of P-gp transfer.

Indeed we did not consider the point (1) since in cancer the case with no cell proliferation does not arise. Point (2) corresponds to Figure 8A\&8C, where cell growth of a two-compartment tumor cell population was plotted without transfer.

7. It is not enough to say that model parameters were "estimated by using intensive parameter estimation computations". Which experimental data were used for model fitting? How was the method of least squares implemented? What weights were used, if any? Also, what are the confidence limits or standard deviations of model parameters? If the confidence intervals are too wide the reported parameter estimates may be misleading.

This point has been detailed.

8. References [22-44]-46] were never mentioned in the text. Am I missing a supplement to the paper?

Corrected.

\section{Technical Comments}

1. P. 7, last line. "... fluorescence $\mathrm{p}$ is assumed to be a function of P-gp expression on the cell surface." It is important to emphasize that this function is strictly increasing.

$O K$, this has been added.

2. P. 9, lines 6-7 from below. Shouldn't the sign of the transfer terms be plus in both cases? Otherwise, if $\mathrm{p} 1<$ p2 then the cell with smaller activity loses it even further while the cell with larger activity gains it.

The signs are correct in the paper, and the part has been carefully explained in the companion paper of Hinow et al.

3. P. 9. What is the biological basis of the two thresh-

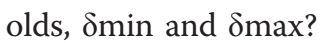

The mentioned thresholds are a conjecture introduced to investigate whether biological data contained constraints in the transferred quantity when fitted with the proposed model. See answer to general comment 3.

4. P. 14, lines 6-7 and p. 18, lines 2-3. Please explain in more detail the interpretation of coefficient $\tau$. Is P-gp transfer thought of as some kind of Poisson process? See also general comment 3.

Yes, the time between transfers is assumed to follow an exponential law. This part is now contained in the paper; see assumption (A2) page 8.

5. P. 15, lines 5-7, Figure $8 \mathrm{~B}$ and p. 26, legend to Figure $8 \mathrm{~B}$. Where does the function $\rho(\mathrm{p})$ displayed in Figure $8 \mathrm{~B}$ come from? Is it determined experimentally?

This function has been established empirically given that it is known that resistance increases as a function of efflux activity which depends on the expression of P-gp (see ref 15, 39 and 40). However, there is no precise study linking the number of P-gp copies at the membrane and the efflux activity. It has to be considered that P-gp pumping is regulated in the minute range, at least by the phosphorylation state of the protein. As a consequence for a given cell at a particular time, a heterogeneous population of P-gps, with pumping activities varying from 0 to a maximal rate, can coexist in the membrane.

6. P. 24, legend to Figure 3, lines 1-2 from below. Where are these "vertical solid lines"?

It was corresponding to an older version of the figure. This sentence has been removed.

7. P. 25, legend to Figure 5. Please explain the role and selection of quadrants in more detail.

This whole legend has been re-written for clarification.

8. P. 25, legend to Figure 6. What are the cell volumes matched to? Give the matching volumes or a reference.

This whole legend has been re-written for clarification. This particular issue is developed in the result section p12 lines 1-5 from below

9. P. 27. Check the estimates for $\delta$ min and $\delta$ max. Shouldn't they be 1.4 and 1.9? What are the units?

It is correct in the paper. The confusion comes from the fact that we used for numerical simulation $\log (p)$ in Figure 6(as well as for the numerical simulations) while in Table 1the result is expressed only using $p$. Figure 6has been corrected.

We thank the referee to point out this problem!

10. P.35, Figure 7. Fluorescence counts are reported on the log scale. Were parameters estimated on the same scale? Changing scale may affect the estimates of model parameters.

See the answer of point 9 above.

\section{Stylistic Comments}

1. P. 5, line 10. The paper denoted [ref] is not specified.

This work is submitted. The given reference is related to an oral communication based on the study.

2. Use a uniform name for the cell line MCF-7/ DOXO. In some places in the text it is called MCF-7/ doxo, see e.g. pp. 6 and 7.

We harmonized the name of cell line to MCF-7/Doxo throughout the text

3. P. 6, line 2 from below. What is S.E.M.?

It is the standard error of the mean, now specified in the text.

4. P. 8. The role and nature of coefficient (or function) h was never explained.

All coefficients, including coefficient $h$, are exposed in table 1 .

The function $h$ has been removed.

5. P. 8, line 7 from below. "describe" should be "describes". 
Ok, this has been changed

6. P. 8, last line. Delete "of" in "transfer of operator T". Ok, this has been deleted

7. P. 14, line 6 from below. What is "volumes of distribution"?

The volume of distribution is a pharmacological concept used to quantify the distribution of a drug between plasma, extracellular fluid, intracellular fluid and other compartments of the body after administration. This concept is useful for studies carried out in vivo.

8. P. 18, lines 4-5 from below. The phrase "biological data as well as modelized transfer parameters suggest an organized quantal trogocytosis of P-gp" is very obscure. What is the meaning of the words "modelized" and "quantal"?

The sentence as been modified as following: In this respect, biological data as well as transfer parameter $f$ suggest an organized quantal transfer of P-gp, in which the minimum unit of exchange would probably consist in a cluster of several copies of the transporter.

9. P. 19, line 4. Shouldn't "administrated" be changed to "administered"?

$\mathrm{Ok}$, this has been changed.

10. P. 19, lines 7-8. What does it mean "separating the injection to the measures"?

In some studies, the effective doxorubicin concentration in the tumor environment is followed after intravenous administration of a dose of the drug. Given that doxorubicin is distributed in several physiological compartments, metabolized and excreted (pharmacokinetics), its concentration in the tumor decreases over time and is all the more low than the time separating the i.v. infusion to the measure is high. The sentence has been slightly modified.

11. P. 20, ref 5. Volume number and pages are missing.

Ok, Volume number and pages have been added.

12. P. 25, line 9. D-E should be D-F.

$O k$, this has been changed.

13. P. 25, line 10. Change "analysis" to "analyses".

$O k$, this has been changed.

The recommendations of the referees were most helpful to us in the improvement of this manuscript.

Reviewer 2: Anna Marciniak-Czochra, University of Heidelberg, Institute of Applied Mathematics, Heidelberg, Germany

The paper is devoted to an interdisciplinary study of the dynamics of cancer cell drug resistance induced by the expression of the drug efflux protein, P-gp. A mathematical model is developed to investigate and validate the hypothesis of cell-to-cell transfer of resistance. The model describes the dynamics of cancer cell population structured by the level of P-gp activity corresponding to the resistance level. It is expressed in the form of a diffusion - drift equation with a nonlocal reaction term describing the transfer between cell subpopulations having different levels of P-gp activity. The model is calibrated and validated based on in vitro experiments with co-cultures of drug-resistant and drug-sensitive human breast carcinoma cells. The model is interesting and shows the evolution of the resistance structure in cancer cell population. Model predictions are compared to experimental observations and biologically relevant conclusions are drawn.

I recommend the manuscript for publication in Biology Direct after minor revisions.

I think that for completeness of paper it would be good to clarify:

1. What is the function $\mathrm{h}(\mathrm{p})$ ?

This question is related to a comment of Reviewer 1: "The paper deals with the model of P-gp transfer with $\varepsilon=\mathrm{h}=0$. Given the predominantly biological readership of the journal, I think it would be better to omit these terms in the model and refer the reader to paper [20] for a more general model accounting for diffusion and drift".

We agree with both referees, and we modified the system accordingly to the comment.

2.Why is spatially homogeneous approach correct? Are there any mixing conditions imposed in the experimental setting? Or it is just a first approach ? Do the authors expect that including spatial effects will influence the results? It would be good to comment on this issue, when model is introduced.

Of course, this is a first approach. From a biological point of view, there is a lot to do in order to give an underlying mechanism for P-gp transfers and several directions have to be explored. Another manuscript will be submitted soon concerning mechanistic. A comment as been added p10 on this issue.

3.How important is the "diffusion" process included in the model? How large is

$\varepsilon$ ? Figure 7 showing that there exist no cells with some small levels of P-gp

activity suggests that such diffusion process is very small, if any.

The term $\varepsilon$ has been removed

\section{Minor remarks}

1. The quality of Figures 4, 6, 8, 9 is not satisfactory, in particular fonts are too small.

Quality of mentioned figures has been enhanced.

Figure 4 is now Figure $3 C D$

Figure 6 is now Figure 5

Figure 8 is now Figure 7

Figure 9 is now Figure 8

2. The description of Figure 3 is confusing; what is (C) and (D)?

It was corresponding to an older version of the figure. This whole legend has been re-written for clarification.

3. On p.5 the reference is missing 
This work is submitted. The given reference is related to an oral communication based on the study.

4. P. 11, line 8 from below: it is written that the peak of activity is shifted to the left towards the region of higher activity. Is it correct? It seems that "left" means "lower activity".

The description is correct. In fact, in these experiments, the cells were loaded with a fluorescent dye that is a substrate of the efflux pump protein. As a consequence, cells expressing a low activity level accumulate intracellular fluorescence and are located in the right part of the graph after analysis, in region of high fluorescence light (FL). On the contrary, cells exhibiting higher activity levels actually efflux the dye and are shifted to the left, a region corresponding to less intracellular fluorescence and thus to higher pumping activity. This is given p12, line 5 from below in the revised version of the manuscript.

Reviewer 3: Marek Kimmel, Houston, United States of America

The paper concerns the important mechanism of horizontal transfer of agents of multiple drug resistance among cancer cells. Based on their own experiments and published data, the authors designed a mathematical model kinetics of which are consistent with the observations. The mathematical model, in the form of a Boltzman-type equation, will serve as a realistic component for models of chemotherapy. The current version of the paper has been improved based on referees' remarks.

\section{Acknowledgements}

This work was supported by grants of the federation FED 4116 SCALE (SCiences Appliquées à L'Environnement). Jennifer Pasquier was a recipient for a fellowship from the Conseil Regional de Haute-Normandie The authors are indebted to Pr. Jean-Pierre Marie (Hôtel Dieu, Paris, France) for providing MCF-7/Doxo and PSC833.

\section{Author details \\ 'Laboratory of Ecotoxicology UPRES EA 3222, IFRMP 23, University of Le Havre, 76058 Le Havre cedex France. 'UMR CNRS 5251 IMB \& INRIA sud- ouest Anubis, Université de Bordeaux, 146 rue Léo Saignat, 33076 Bordeaux, France. ${ }^{3}$ Department of Mathematics, Vanderbilt University, 1326 Stevenson Center, Nashville, TN37240, USA.}

\section{Authors' contributions}

JP carried out cell cultures, confocal imaging, flow cytometry analysis and drafted the manuscript. PM developed the math model and computed the simulation. CB-L participated to the design of biological experiments and drafted the manuscript. GW participated to the design of the math model and helped to draft the manuscript. FLF coordinated the study and wrote the manuscript. All authors read and approved the final manuscript.

\section{Competing interests}

The authors declare that they have no competing interests.

Received: 8 April 2010 Accepted: 26 January 2011

Published: 26 January 2011

\section{References}

1. Blagosklonny MV: Molecular theory of cancer. Cancer Biol Ther 2005, 4:621-7.

2. Hanahan D, Weinberg RA: The hallmarks of cancer. Cell 2000, 100:57-70.
3. Mueller MM, Fusenig NE: Friends or foes - bipolar effects of the tumour stroma in cancer. Nat Rev Cancer 2004, 4:839-49.

4. Axelrod R, Axelrod DE, Pienta KJ: Evolution of cooperation among tumor cells. Proc Natl Acad Sci USA 2006, 103:13474-9.

5. Coley HM: Mechanisms and strategies to overcome chemotherapy resistance in metastatic breast cancer. Cancer Treat Rev 2008, 34:378-390.

6. Greenberg PA, Hortobagyi GN, Smith TL, Ziegler LD, Frye DK, Buzdar AU: Long-term follow-up of patients with complete remission following combination chemotherapy for metastatic breast cancer. J Clin Oncol 1996, 14:2197-205

7. Bonneterre J, Dieras $\mathrm{V}$, Tubiana-Hulin M, Bougnoux P, Bonneterre ME, Delozier T, Mayer F, Culine S, Dohoulou N, Bendahmane B: Phase II multicentre randomised study of docetaxel plus epirubicin vs 5fluorouracil plus epirubicin and cyclophosphamide in metastatic breast cancer. Br J Cancer 2004, 91:1466-71.

8. Longley DB, Johnston PG: Molecular mechanisms of drug resistance. J Pathol 2005, 205:275-92.

9. Broxterman HJ, Gotink KJ, Verheul HM: Understanding the causes of multidrug resistance in cancer: a comparison of doxorubicin and sunitinib. Drug Resist Updat 2009, 12:114-26.

10. Sparreboom A, Danesi R, Ando Y, Chan J, Figg WD: Pharmacogenomics of $A B C$ transporters and its role in cancer chemotherapy. Drug Resistance Updates 2003, 6:71-84.

11. Dano K: Cross resistance between vinca alkaloids and anthracyclines in Ehrlich ascites tumor in vivo. Cancer Chemother Rep 1972, 56:701-8.

12. Juliano RL, Ling $V$ : A surface glycoprotein modulating drug permeability in Chinese hamster ovary cell mutants. Biochim Biophys Acta 1976, 455:152-62.

13. Trock BJ, Leonessa F, Clarke R: Multidrug resistance in breast cancer: a meta-analysis of MDR1/gp170 expression and its possible functional significance. J Natl Cancer Inst 1997, 89:917-31.

14. Leonessa F, Clarke R: ATP binding cassette transporters and drug resistance in breast cancer. Endocr Relat Cancer 2003, 10:43-73.

15. Broxterman HJ, Sonneveld P, Feller N, Ossenkoppele GJ, Wahrer DC, Eekman CA, Schoester M, Lankelma J, Pinedo HM, Lowenberg B, et al: Quality control of multidrug resistance assays in adult acute leukemia: correlation between assays for P-glycoprotein expression and activity. Blood 1996, 87:4809-16.

16. Meesungnoen J, Jay-Gerin JP, Mankhetkorn S: Relation between MDR1 mRNA levels, resistance factor, and the efficiency of P-glycoproteinmediated efflux of pirarubicin in multidrug-resistant K562 sublines. Can J Physiol Pharmacol 2002, 80:1054-63.

17. Linn SC, Giaccone G, van Diest PJ, Blokhuis WM, van der Valk P, van Kalken CK, Kuiper CM, Pinedo HM, Baak JP: Prognostic relevance of P-glycoprotein expression in breast cancer. Ann Oncol 1995, 6:679-85.

18. Licht T, Fiebig HH, Bross KJ, Herrmann F, Berger DP, Shoemaker R, Mertelsmann R: Induction of multiple-drug resistance during antineoplastic chemotherapy in vitro. Int J Cancer 1991, 49:630-7.

19. Hu XF, Slater A, Wall DM, Kantharidis P, Parkin JD, Cowman A, Zalcberg JR: Rapid up-regulation of mdr1 expression by anthracyclines in a classical multidrug-resistant cell line. Br J Cancer 1995, 71:931-6.

20. Molinari A, Toccacieli L, Calcabrini A, Diociaiuti M, Cianfriglia M, Arancia G Induction of P-glycoprotein expression on the plasma membrane of human melanoma cells. Anticancer Res 2000, 20:2691-6.

21. Pesic M, Markovic JZ, Jankovic D, Kanazir S, Markovic ID, Rakic L, Ruzdijic S Induced resistance in the human non small cell lung carcinoma ( $\mathrm{NCl}$ H460) cell line in vitro by anticancer drugs. J Chemother 2006, 18:66-73.

22. Luu KT, Uchizono JA: P-glycoprotein induction and tumor cell-kill dynamics in response to differential doxorubicin dosing strategies: a theoretical pharmacodynamic model. Pharm Res 2005, 22:710-5.

23. Levchenko A, Mehta BM, Niu X, Kang G, Villafania L, Way D, Polycarpe D, Sadelain M, Larson SM: Intercellular transfer of P-glycoprotein mediates acquired multidrug resistance in tumor cells. Proc Natl Acad Sci USA 2005, 102:1933-8.

24. Rafii A, Mirshahi P, Poupot M, Faussat AM, Simon A, Ducros E, Mery E, Couderc B, Lis R, Capdet J, et al: Oncologic trogocytosis of an original stromal cells induces chemoresistance of ovarian tumours. PLOS ONE 2008, 3:e3894.

25. Bebawy M, Combes V, Lee E, Jaiswal R, Gong J, Bonhoure A, Grau GE: Membrane microparticles mediate transfer of P-glycoprotein to drug sensitive cancer cells. Leukemia 2009, 23:1643-9. 
26. Hinow $P$, Le Foll F, Magal P, Webb GF: Analysis of a model for transfer phenomena in biological populations. SIAM Journal of applied mathematics 2009, 70:40-62.

27. Pasquier J, Galas L, Boulangé-Leconte C, Bultelle F, Magal P, Webb GF, Le Foll F: Tunneling nanotubes and microparticles as concurrent carriers for cell-to-cell P-glycopretein transfers: evidence for extragenetic acquired resistance in breast cancer cells. Workshop: progress in cellular imaging Rouen; 2010

28. Marin M, Poret A, Maillet $G$, Leboulenger F, Le Foll F: Regulation of volume-sensitive $\mathrm{Cl}$ - channels in multi-drug resistant MCF7 cells. Biochem Biophys Res Commun 2005, 334:1266-78.

29. Zhou Y, Gottesman MM, Pastan I: The extracellular loop between TM5 and TM6 of P-glycoprotein is required for reactivity with monoclonal antibody UIC2. Arch Biochem Biophys 1999, 367:74-80.

30. Frankfurt OS, Seckinger D, Sugarbaker EV: Intercellular transfer of drug resistance. Cancer Res 1991, 51:1190-5.

31. Hennessy M, Spiers JP: A primer on the mechanics of P-glycoprotein the multidrug transporter. Pharmacol Res 2007, 55:1-15.

32. Beck WT, Grogan TM, Willman CL, Cordon-Cardo C, Parham DM, Kuttesch JF, Andreeff M, Bates SE, Berard CW, Boyett JM, et al: Methods to detect P-glycoprotein-associated multidrug resistance in patients tumors: consensus recommendations. Cancer Res 1996, 56:3010-20.

33. Faneyte IF, Kristel PM, van de Vijver MJ: Determining MDR1/P-glycoprotein expression in breast cancer. Int J Cancer 2001, 93:114-22.

34. Sanfilippo O, Ronchi E, De Marco C, Di Fronzo G, Silvestrini R: Expression of P-glycoprotein in breast cancer tissue and in vitro resistance to doxorubicin and vincristine. Eur J Cancer 1991, 27:155-8.

35. Li EX, Li R, Zhang ZH, Wang JB: Clinical significance of P-glycoprotein expression in breast cancer. Chinese Journal of Cancer Research 1999, 11:218-220.

36. Arnal M, Franco N, Fargeot $P$, Riedinger JM, Brunet-Lecomte $P$, LizardNacol S: Enhancement of mdr1 gene expression in normal tissue adjacent to advanced breast cancer. Breast Cancer Res Treat 2000, 61:13-20.

37. Allen JD, Brinkhuis RF, van Deemter L, Wijnholds J, Schinkel AH: Extensive contribution of the multidrug transporters P-glycoprotein and Mrp1 to basal drug resistance. Cancer Res 2000, 60:5761-6.

38. Chaudhary PM, Mechetner EB, Roninson IB: Expression and activity of the multidrug resistance $\mathrm{P}$-glycoprotein in human peripheral blood lymphocytes. Blood 1992, 80:2735-9.

39. Ferrand VL, Montero Julian FA, Chauvet MM, Hirn MH, Bourdeaux MJ: Quantitative determination of the MDR-related P-glycoprotein, Pgp 170, by a rapid flow cytometric technique. Cytometry 1996, 23:120-5.

40. Oleinikov VA, Fleury F, lanoul A, Zaitsev S, Nabiev I: P-glycoprotein effect on the properties of its natural lipid environment probed by Raman spectroscopy and Langmuir-Blodgett technique. FEBS Lett 2006 , 580:4953-8.

41. Carlson RW, Anderson BO, Burstein HJ, Carter WB, Edge SB, Farrar WB, Goldstein LJ, Gradishar WJ, Hayes DF, Hudis CA, et al: Invasive breast cancer. J Natl Compr Canc Netw 2007, 5:246-312.

42. Rossi C, Gasparini G, Canobbio L, Galligioni E, Volpe R, Candiani E, Toffoli G, D'Incalci M: Doxorubicin distribution in human breast cancer. Cancer Treat Rep 1987, 71:1221-6.

43. Speth PA, Linssen PC, Holdrinet RS, Haanen C: Plasma and cellular adriamycin concentrations in patients with myeloma treated with ninety-six-hour continuous infusion. Clin Pharmacol Ther 1987, 41:661-5.

44. Laginha KM, Verwoert S, Charrois GJ, Allen TM: Determination of doxorubicin levels in whole tumor and tumor nuclei in murine breast cancer tumors. Clin Cancer Res 2005, 11:6944-9.

doi:10.1186/1745-6150-6-5

Cite this article as: Pasquier et al:: Consequences of cell-to-cell P-

glycoprotein transfer on acquired multidrug resistance in breast cancer: a cell population dynamics model. Biology Direct 2011 6:5.

\section{Submit your next manuscript to BioMed Central and take full advantage of:}

- Convenient online submission

- Thorough peer review

- No space constraints or color figure charges

- Immediate publication on acceptance

- Inclusion in PubMed, CAS, Scopus and Google Scholar

- Research which is freely available for redistribution

Submit your manuscript at www.biomedcentral.com/submit 